\title{
THE DERIVATIVE OF AN INCOHERENT EISENSTEIN SERIES II
}

\author{
Hui Xue
}

\begin{abstract}
Let $K$ be an imaginary quadratic field with the norm map $\mathbf{N}$. Let $(K,-\kappa \mathbf{N})$ be a binary quadratic form with $\kappa$ a positive rational number. In this paper, under some minor conditions on $\kappa$, we first construct an incoherent Eisenstein series (in the sense of Kudla) associated to $(K,-\kappa \mathbf{N})$. Then we study its derivative at the center of symmetry $s=0$, and show that each non-constant Fourier coefficient of the derivative can be interpreted as the degree of certain zero-dimensional schemes. This result together with our previous work gives a complete answer to a question raised by Kudla-Rapoport-Yang.
\end{abstract}

\section{Introduction}

Let $K=\mathbb{Q}(\sqrt{-D})$ be an imaginary quadratic field of discriminant $-D$. Let $B$ be an indefinite division quaternion algebra over $\mathbb{Q}$ of discriminant $N$. We assume that $K$ can be embedded into $B$ and fix such an embedding $\imath: K \rightarrow B$. We also assume that $N$ is relatively prime to $D$. The embedding $\iota$ induces an orthogonal decomposition with respect to the norm: $\left(B, \mathbf{N}_{B}\right)=(K, \mathbf{N}) \oplus(K,-\kappa \mathbf{N})$ with $\kappa \in \mathbb{Q}^{\times}$. Our assumption on $B$ and $K$ implies that $(-D, \kappa)_{p}=-1$ if $p \mid N$, and $(-D, \kappa)_{p}=1$ if $p \nmid N$. In particular $\kappa>0$. Here $(\cdot, \cdot)_{p}$ is the local Hilbert symbol at the (finite or infinite) prime $p$.

In Section 2, following the general mechanism developed by Kudla, we will define a modified incoherent Eisenstein series $\mathcal{E}(g, s, \Phi)$ on $S L_{2}(\mathbb{A})$ which is constructed from an incoherent collection $\mathcal{C}=\left\{\mathcal{C}_{p}\right\}_{p}$ of local binary quadratic spaces. This incoherent collection $\mathcal{C}$ coincides with the local quadratic space $\left(K_{l},-\kappa \mathbf{N}\right)$ at every finite prime $l$, except that $\mathcal{C}_{\infty}=\left(K_{\infty}, \kappa \mathbf{N}\right)$. The Eisenstein series $\mathcal{E}(g, s, \Phi)$ vanishes at the center of symmetry $s=0$. Let $\tau=u+i v$ be in the upper half plane, write $g_{\tau}=\left(\begin{array}{ll}1 & u \\ 0 & 1\end{array}\right)\left(\begin{array}{cc}v^{1 / 2} & 0 \\ 0 & v^{-1 / 2}\end{array}\right)$, and define

$$
\phi(\tau)=-\left.\frac{d}{d s}\left(v^{-1 / 2} \mathcal{E}\left(g_{\tau}, s, \Phi\right)\right)\right|_{s=0} .
$$

Then $\phi(\tau)$ is a non-holomorphic modular form of weight 1. Let

$$
\phi(\tau)=a_{0}(v)+\sum_{t>0} a_{t} e^{2 \pi i \tau}+\sum_{t<0} a_{t}(v) e^{2 \pi i \tau}
$$

be its Fourier expansion. In this paper, we will study the Fourier coefficients of $\phi(\tau)$, and show for $t>0$ the following identity

$$
a_{t}=\left(2^{d} w_{K}\right)^{-1} \operatorname{deg}(Z(t)),
$$

Received by the editors October 4, 2011.

2010 Mathematics Subject Classification. 11F30, 11F37, 11G40. 
where $Z(t)$ is a zero-dimensional scheme supported only over primes which are inert or ramified in $K$, see Theorem 3.2 of Section 3.3 for the precise statement and interpretations of other Fourier coefficients.

We will devote the rest of this introduction to a brief description of the idea to prove (1.1) and the relation with our previous paper [11]. We basically follow the same approach as that in [11]. In [11], the splitting case is considered, i.e., the quaternion algebra $B$ is the splitting algebra of $2 \times 2$ matrices. In the splitting case, if $D$ is prime the identity (1.1) was first proved by Kudla-Rapoport-Yang [7].

In [11], we find a different approach and managed to give a simplified proof of (1.1) for all fundamental discriminants $D$. In that paper, we do not compute either side of (1.1) as explicitly as the authors did in [7]. Instead, after some light computation we reduce the left-hand side of (1.1) to a weighted sum over non-split primes $p$ of the $t$-th Fourier coefficients of various coherent Eisenstein series $E^{*}\left(g, s, \Phi^{(p)}\right)$. Then we show that each weight matches the length of the local ring $\mathcal{O}_{Z(t), \xi}$ at any geometric point $\xi$ of $Z(t)$ over $\overline{\mathbb{F}}_{p}$. By applying a Siegel-Weil formula we also show that the $t$-th Fourier coefficient of $E^{*}\left(g, s, \Phi^{(p)}\right)$ equals the number of geometric points of $Z(t)$ over $\overline{\mathbb{F}}_{p}$. As the degree of $Z(t)$ is given by the weighted sum of lengths of local rings at all geometric points over $\overline{\mathbb{F}}_{p}$ for various $p$, we are finally able to derive the equality (1.1). We learned from the referee that in [9], using a method similar to that of [11], Kudla and Yang have independently proved (1.1) (and much more) for general odd discriminants $D$ (but still in the splitting case).

The present paper serves as a complement to [11]. The case studied in this paper is a non-splitting one, i.e., $B$ is assumed to be a division algebra. So new phenomenon naturally presents at the primes dividing $N$, the discriminant of $B$. At these places, the $t$-th Fourier coefficient of the derivative of the original incoherent Eisenstein series $E^{*}(g, s, \Phi)$ defined in [11] does not quite match $\operatorname{deg}(Z(t))$. To remedy the discrepancy we need to modify the definition to get the so called modified incoherent Eisenstein series $\mathcal{E}(g, s, \Phi)$. Roughly speaking, the series $\mathcal{E}(g, s, \Phi)$ is obtained by adding a suitable combination of certain coherent Eisenstein series $E^{*}\left(g, s, \Psi^{(p)}\right)$ for all $p \mid N$, see (2.10) for the detail. The idea of modifying Eisenstein series was first introduced by Kudla-Rapoport-Yang [8]. In [8] the authors studied the derivative at $s=1 / 2$ of a modified coherent Eisenstein series of weight $3 / 2$ associated to a division quaternion algebra $B$. The result of [8] can be viewed as the one-dimensional counterpart of our zero-dimensional case, so it seems natural for us to borrow the idea therein. Another difference between the present paper and [11] is that, on the geometric side, in this paper QM abelian surfaces with $\mathrm{CM}$ by $\mathcal{O}_{K}$ need to be studied, while in [11] only elliptic curves with CM by $\mathcal{O}_{K}$ are considered.

Notation. The letter $G$ denotes the group $S L_{2}$. The standard Borel subgroup of $S L_{2}$ is denoted by $B$. The following notation is used for elements in $S L_{2}$ :

$$
m(a)=\left(\begin{array}{cc}
a & 0 \\
0 & a^{-1}
\end{array}\right), \quad n(b)=\left(\begin{array}{ll}
1 & b \\
0 & 1
\end{array}\right), \quad w=\left(\begin{array}{cc}
0 & 1 \\
-1 & 0
\end{array}\right) .
$$

The ring of adeles of $\mathbb{Q}$ is denoted by $\mathbb{A}$. The letter $\omega$ denotes the quadratic character of the idele class group $\mathbb{A}^{\times} / \mathbb{Q}^{\times}$associated to $K=\mathbb{Q}(\sqrt{-D})$. For an algebraic object $L$ we write $\widehat{L}=L \otimes \widehat{\mathbb{Z}}$ and write $1_{L}$ for the characteristic function of the set $L$. For $g \in$ 
$G(\mathbb{A})$, let $g=n(b) m(a) k$ be its Iwasawa decomposition, where $k \in S O_{2}(\mathbb{R}) S L_{2}(\widehat{\mathbb{Z}})$. Then the absolute value $|a(g)|=|a|_{\mathbb{A}}$ is independent of the decomposition.

The additive character $\psi$ of $\mathbb{A} / \mathbb{Q}$ is the standard one defined in Tate's thesis such that $\psi_{\infty}(x)=e^{2 \pi i x}$.

\section{Analytic computations}

In this section, we will first quickly recall the definition of various (coherent or incoherent) Eisenstein series. Then we define the modified Eisenstein series and calculate the Whittaker values of the derivative $\mathcal{E}^{\prime}\left(g_{\tau}, 0, \Phi\right)$ at $s=0$.

2.1. Quadratic spaces and Schwartz functions. Let $B$ be the indefinite quaternion algebra and $K=\mathbb{Q}(\sqrt{-D})$ be the imaginary quadratic field fixed as in Section 1 . We denote the quadratic character associated to $K$ by $\omega$. Define

$$
V=\{y \in B \mid \iota(a) \cdot y=y \cdot \iota(\bar{a}), \text { for all } a \in K\},
$$

and let $q$ be the quadratic norm induced by $\mathbf{N}_{B}$. So $(V, q) \cong(K,-\kappa \mathbf{N})$, and we have the orthogonal decomposition of quadratic spaces

$$
\left(B, \mathbf{N}_{B}\right)=(K, \mathbf{N}) \oplus(V, q) .
$$

From now on we always let $\mathcal{O}_{B}$ be a fixed maximal order of $B$ which contains $\mathcal{O}_{K}$. The incoherent collection $\mathcal{C}=\left\{\mathcal{C}_{p}\right\}$ is taken to be the collection of local quadratic spaces, such that at each finite prime $p \mathcal{C}_{p}=\left(V_{p}, q_{p}\right) \cong\left(K_{p},-\kappa \mathbf{N}\right)$ and $\mathcal{C}_{\infty}=\left(V_{\infty},-q_{\infty}\right) \cong(\mathbb{C}, \kappa \mathbf{N})$. We now defined a special element $\varphi=\otimes_{p} \varphi_{p}$ in the space of Schwartz functions $S\left(\mathcal{C}_{\mathbb{A}}\right)$ on $\mathcal{C}$. Let $L=V \cap \mathcal{O}_{B}$ be the quadratic lattice in $V$. For each prime $p<\infty$, the local Schwartz function $\varphi_{p}$ is the characteristic function $1_{L_{p}}$ of $L \otimes \mathbb{Z}_{p}$. At $p=\infty$, we take $\varphi_{\infty}=e^{-2 \pi \kappa \mathbf{N}(z)}$ on $V_{\infty}=\mathbb{C}$.

For each prime $p$ inert or ramified in $K$, we will define a (coherent) rational quadratic space $V^{(p)}$ and a Schwartz function $\varphi^{(p)}=\otimes_{l} \varphi_{l}^{(p)}$ in $S\left(V_{\mathbb{A}}\right)$. Let us begin with finite $p$. Assume $(A, \imath)$ is a $\mathrm{QM}$ abelian surface over $\overline{\mathbb{F}}_{p}$ with $\mathrm{CM}$ by the embedding $\iota: \mathcal{O}_{K} \rightarrow \operatorname{End}(A, \imath)$, see Section 3 for the definition of QM abelian surfaces. Thus $(A, \imath)$ is supersingular and $\mathcal{O}^{(p)}=\operatorname{End}(A, \imath)$ is an order of discriminant $p N$ in the definite quaternion algebra $B^{(p)}$ of discriminant $N / p$. Let

$$
V^{(p)}=\left\{y \in B^{(p)} \mid \iota(a) \cdot y=y \cdot \iota(\bar{a}), \text { for all } a \in K\right\},
$$

then we have the following orthogonal decomposition of quadratic spaces

$$
\operatorname{End}(A, \imath)=B^{(p)}=K \oplus V^{(p)} .
$$

Write

$$
L^{(p)}=\mathcal{O}^{(p)} \cap V^{(p)},
$$

so $L^{(p)}$ is a quadratic lattice in $V^{(p)}$. Now the local Schwartz function $\varphi_{l}^{(p)}$ for each finite prime $l$ is defined to be the characteristic function $1_{L_{l}^{(p)}}$ of the local lattice $L_{l}^{(p)}=L^{(p)} \otimes \mathbb{Z}_{l}$. At $l=\infty$, suppose $\left(V_{\infty}^{(p)}, \mathbf{N}\right) \cong(\mathbb{C}, \Lambda \mathbf{N})$, then

$$
\varphi_{\infty}^{(p)}(z)=e^{-2 \pi|\Lambda| \mathbf{N}(z)} .
$$


Now suppose $p=\infty$ and let $(A, \imath, \iota)$ be a fixed QM abelian surface over $\mathbb{C}$ with $\mathrm{CM}$ by $\mathcal{O}_{K}$. Similarly, the embedding $\iota: \mathcal{O}_{K} \rightarrow \mathcal{O}^{(\infty)}=\operatorname{End}\left(A^{a}, \imath\right) \cong \mathcal{O}_{B}^{\text {opp }}$ (see Section 3 for more details) induces an orthogonal decomposition

$$
B^{(\infty)} \cong B^{\text {opp }}=K \oplus V^{(\infty)},
$$

where $V^{(\infty)}=\left\{y \in B^{(\infty)} \mid \iota(a) \cdot y=y \cdot \iota(\bar{a})\right\}$. Let

$$
L^{(\infty)}=\mathcal{O}^{(\infty)} \cap V^{(\infty)}
$$

be a quadratic lattice in $V^{(\infty)}$. For each prime $l<\infty$ the local Schwartz function $\varphi_{l}^{(\infty)}$ is then defined to be the characteristic function of $L_{l}^{(\infty)}$, and $\varphi_{\infty}^{(\infty)}(z)=e^{-2 \pi|\Lambda| \mathbf{N}(z)}$ provided that $\left(V_{\infty}^{\infty}, \mathbf{N}\right)=(\mathbb{C}, \Lambda \mathbf{N})$.

In order to define the modified incoherent Eisenstein series in next section, for each $p \mid N$ we also need to define another collection of local Schwartz functions $\left\{\psi_{l}^{(p)}\right\}$. This time we let $\mathcal{O}_{B^{(p)}}$ be a maximal order of $B^{(p)}$, and define a new quadratic lattice

$$
M^{(p)}=\mathcal{O}_{B(p)} \cap V^{(p)} .
$$

For each $l<\infty$ the local Schwartz function $\psi_{l}^{(p)}$ is the characteristic function of the local lattice $M_{l}^{(p)}=M^{(p)} \otimes \mathbb{Z}_{l}$. At $\infty$ the function $\psi_{\infty}^{(p)}$ is given exactly in the same way as $\varphi_{\infty}^{(p)}$.

2.2. Eisenstein series. Let $(V, q)$ be binary quadratic space over a local field $F$. Let $S O(V)$ be the group defined by

$$
S O(V)=\left\{\sigma \in G L_{F}(V): q(\sigma v)=q(v) \text { for } v \in V\right\} .
$$

Given a fixed additive character $\psi$ of $F$ the Weil representation $r_{V}=r_{(V, q), \psi}$ of $S L_{2}(F)$ on the space $S(V)$ of Schwartz functions on $V$ is defined by the following rules (see [4])

$$
\begin{aligned}
r_{V}\left(\left(\begin{array}{ll}
1 & a \\
0 & 1
\end{array}\right)\right) f(x) & =\psi(a q(x)) f(x), \\
r_{V}\left(\left(\begin{array}{cc}
a & 0 \\
0 & a^{-1}
\end{array}\right)\right) f(x) & =|a| \omega_{V}(a) f(a x), \\
r_{V}\left(\left(\begin{array}{cc}
0 & 1 \\
-1 & 0
\end{array}\right)\right) f(x) & =\gamma_{V} \hat{f}(x),
\end{aligned}
$$

where $\gamma_{V}$, called the Weil index of $V$, is an eighth root of unity, $\omega_{V}$ is the quadratic character of $F^{\times}$associated to the quadratic space $V$, which is defined by $\omega_{V}(x)=$ $\left(x,-d_{V}\right)$, where $d_{V}$ is the determinant of $V$ and $(\cdot, \cdot)$ is the Hilbert symbol over $F$. The measure on $V$ is taken to be self-dual with respect to the Fourier transform $\hat{f}(x)=\int_{V} f(y) \psi(q(x, y)) d y$, where $q(x, y)=q(x+y)-q(x)-q(y)$. In particular, if $(V, q)=(K, \mathbf{N})$ and $F$ is non-archimedean, then the total measure of $\mathcal{O}_{K}$ is $\left|d_{K / F}\right|^{1 / 2}$, where $d_{K / F}$ is the relative discriminant of $K / F$. If $F$ is archimedean, that is $K=\mathbb{C}$, then the measure on $K$ is given by $2 d x d y$.

Let

$$
I(s, \omega)=\operatorname{Ind}_{B(\mathbb{A})}^{G(\mathbb{A})}\left(\omega|\cdot|^{s}\right)
$$

be the global induced representation of $G(\mathbb{A})$. Each $\Phi(g, s) \in I(s, \omega)$ thus satisfies

$$
\Phi(n(b) m(a) g, s)=\omega(a)|a|^{s+1} \Phi(g, s) .
$$


At $s=0$, the induced representation $I(0, \omega)$ has the following decomposition into irreducible representations of $G(\mathbb{A})$ :

$$
I(0, \omega)=\left(\oplus_{V} \Pi(V)\right) \oplus\left(\oplus_{\mathcal{C}} \Pi(\mathcal{C})\right) .
$$

Here $V$ runs over rational binary quadratic spaces with $\omega_{V}=\omega$, and $\mathcal{C}$ runs through incoherent collections (in the sense of Kudla [6]) of local binary quadratic spaces with $\omega_{\mathcal{C}}=\omega$. The subspace $\Pi(V)$ is the image of the equivariant map $\lambda_{V}: S\left(V_{\mathbb{A}}\right) \rightarrow I(0, \omega)$ defined by

$$
\lambda_{V}\left(\otimes_{p} \varphi_{p}\right)(g)=\otimes_{p}\left(\left(r_{V, p}(g) \varphi_{p}\right)(0) .\right.
$$

Similarly, $\Pi(\mathcal{C})$ is the image of the equivariant map $\lambda_{\mathcal{C}}: S\left(\mathcal{C}_{\mathbb{A}}\right) \rightarrow I(0, \omega)$ defined by

$$
\lambda_{\mathcal{C}}\left(\otimes_{p} \varphi_{p}\right)(g)=\otimes_{p}\left(\left(r_{\mathcal{C}_{p}}(g) \varphi_{p}\right)(0)\right.
$$

For each prime $p$ not split in $K$, we define a coherent standard section by

$$
\Phi^{(p)}=\otimes_{l} \Phi_{l}^{(p)}=\lambda_{V^{(p)}}\left(\varphi^{(p)}\right) \in I(0, \omega) .
$$

At each $p \mid N$, another coherent standard section is defined by

$$
\Psi^{(p)}=\otimes_{l} \Psi_{l}^{(p)}=\lambda_{V^{(p)}}\left(\psi^{(p)}\right) \in I(0, \omega) .
$$

Similarly the incoherent section is given by

$$
\Phi=\otimes_{l} \Phi_{l}=\lambda_{\mathcal{C}}\left(\varphi_{l}\right) \in I(0, \omega) .
$$

The next lemma is taken from [11, Lemma 2.3] which compares local canonical sections attached to various quadratic spaces.

Lemma 2.1. If a (finite or infinite) prime l is different from $p$, then $\Phi_{l}^{(p)}=\Psi_{l}^{(p)}=\Phi_{l}$.

Now we are ready to define various Eisenstein series. Let us begin with the incoherent ones. Let $\Phi(g) \in \Pi(\mathcal{C}) \subset I(0, \omega)$ be as above, and set

$$
\Phi(g, s)=\Phi(g)|a(g)|^{s}
$$

be the standard extension to $I(s, \omega)$. The incoherent Eisenstein series is given by

$$
E(g, s, \Phi)=\sum_{\gamma \in B \backslash G} \Phi(\gamma g, s)
$$

converges absolutely for $\operatorname{Re}(s)>1$. The series $E(g, s, \Phi)$ has weight 1 at infinity [10], and it has an analytic continuation so that $[6]$

$$
E(g, 0, \Phi)=0 \text {. }
$$

We complete the Eisenstein series by a factor

$$
E^{*}(g, s, \Phi)=D^{(s+1) / 2} \Lambda(s+1, \omega) \cdot E(g, s, \Phi),
$$

where $\Lambda(s, \omega)=\prod_{p \leq \infty} L\left(s, \omega_{p}\right)$ is the complete $L$-function of the character $\omega$.

Similarly, for each prime $p$ non-split in $K$, the (coherent) Eisenstein series associated to $\Phi^{(p)}$ is defined by

$$
E\left(g, s, \Phi^{(p)}\right)=\sum_{\gamma \in B \backslash G} \Phi^{(p)}(\gamma g, s) .
$$

with

$$
\Phi^{(p)}(g, s)=\Phi^{(p)}(g)|a(g)|^{s} .
$$


The complete coherent Eisenstein series is given by

$$
E^{*}\left(g, s, \Phi^{(p)}\right)=D^{(s+1) / 2} \Lambda(s+1, \omega) \cdot E\left(g, s, \Phi^{(p)}\right) .
$$

In a similar fashion, for each prime $p \mid N$, we define $E\left(g, s, \Psi^{(p)}\right)$ and its completion $E^{*}\left(g, s, \Psi^{(p)}\right)$.

To treat the primes at which $B$ is ramified, i.e., the primes dividing $N$, we need to modify the complete incoherent Eisenstein series $E^{*}(g, s, \Phi)$. First, for each prime $p \mid N$, we fix an auxiliary function $c_{p}(s)$, which is a rational function of $p^{-s}$, such that

$$
c_{p}(0)=0 \quad \text { and } \quad c_{p}^{\prime}(0)=\frac{1}{1+p} \log p,
$$

and such that

$$
c_{p}(s)=-c_{p}(-s) .
$$

The modified Eisenstein series is then defined as

$$
\mathcal{E}(\tau, s, \Phi)=E^{*}(\tau, s, \Phi)+\sum_{p \mid N} c_{p}(s) E^{*}\left(\tau, s, \Psi^{(p)}\right) .
$$

The modified series $\mathcal{E}(g, s, \Phi)$ can be analytically continued to the whole complex plane (as each term involved can), and by the exact definition of $c_{p}(s)$ :

$$
\mathcal{E}(g, 0, \Phi)=0 \text {. }
$$

Remark 2.1. (1) There are many choices for such $c_{p}(s)$. For instance, we can take

$$
c_{p}(s)=\frac{1}{2(1+p)}\left(p^{s}-p^{-s}\right) .
$$

(2) The idea of modifying a standard Eisenstein series is inspired by [8, Section 6$]$. In [8], the authors modified a incoherent Eisenstein series by adding a combination of incoherent ones, while here we modify an incoherent Eisenstein series with a combination of coherent ones.

(3) The choice of $c_{p}(s)$ is made such that $\mathcal{E}(g, s, \Phi)$ satisfies an odd functional equation under $s \mapsto-s$.

2.3. Whittaker integrals. In this section, we will carry out the analytic computation of the Fourier coefficients of the central derivative $\mathcal{E}^{\prime}(g, 0, \Phi)$. In the range of absolute convergence $\operatorname{Re}(s)>1$, the Eisenstein series $E(g, s, \Phi)$ has a Fourier expansion

$$
E(g, s, \Phi)=\sum_{t \in \mathbb{Q}} E_{t}(g, s, \Phi)
$$

with

$$
E_{t}(g, s, \Phi)=\int_{\mathbb{Q} \backslash \mathbb{A}} E(n(b) g, s, \Phi) \psi(-t b) d b .
$$

It is easy to see that $E_{t}(g, s, \Phi)=0$ if $t \notin \mathbb{Z}$ (see [7, Lemma 2.3]). So from now on we always assume that $t$ is an integer.

For $t \neq 0$ unfolding (2.11) gives

$$
E_{t}(g, s, \Phi)=\prod_{p} W_{t, p}\left(g_{p}, s, \Phi_{p}\right),
$$


with

$$
W_{t, p}\left(g_{p}, s, \Phi_{p}\right)=\int_{\mathbb{Q}_{p}} \Phi_{p}\left(w n(b) g_{p}, s\right) \psi_{p}(-t b) d b
$$

being called a local Whittaker integral. For $t=0$ the constant term is given by

$$
E_{0}(g, s, \Phi)=\Phi(g, s)+M(s) \Phi(g),
$$

where $M(s) \Phi=\otimes_{p} M_{p}(s) \Phi_{p}$ is the intertwining map from $I(s, \omega)$ to $I(-s, \omega)$, and

$$
M_{p}(s) \Phi_{p}(g)=W_{0, p}\left(g, s, \Phi_{p}\right) .
$$

The modified local Whittaker integral is defined as

$$
W_{t, p}^{*}\left(g, s, \Phi_{p}\right)=L_{p}\left(s+1, \omega_{p}\right) \cdot W_{t, p}\left(g, s, \Phi_{p}\right) .
$$

All the above constructions and definitions also apply to $E\left(g, s, \Phi^{(p)}\right)$ and will be assumed automatically.

To get the Fourier coefficients of $\mathcal{E}^{\prime}(g, s, \Phi)$ we need to compute the local Whittaker integrals $W_{t, p}^{*}(g, s, \Phi)$ for $g=1$ at $p<\infty$ and for

$$
g_{\tau}=\left(\begin{array}{ll}
1 & u \\
0 & 1
\end{array}\right)\left(\begin{array}{cc}
v^{1 / 2} & 0 \\
0 & v^{-1 / 2}
\end{array}\right)=n(u) m\left(v^{1 / 2}\right),
$$

at $p=\infty$.

For each prime $p$ not split in $K$, we introduce the following notation. Let $d_{p}=v_{p}(D)$ and let $f_{p}$ be the residue degree of $K / \mathbb{Q}$ at the prime $p$. For each non-zero integer $t$ define

$$
\ell_{p}(t)= \begin{cases}\frac{v_{p}(t)+1}{2}, & \text { if } p \text { is inert in } K \text { and } p \nmid N, \\ \frac{v_{p}(t)}{2}, & \text { if } p \mid N, \\ v_{p}(t)+d_{p}, & \text { if } p \text { is ramified in } K .\end{cases}
$$

See Proposition 3.1 for justification of the above convention.

We first recall some results from [11] on the local Whittaker integrals at places not dividing $N$.

Proposition 2.1. Let $t \neq 0$ and $p \nmid N$ be not split in $K$, then $W_{t, p}^{*}(1,0, \Phi)=0$ if and only if $\omega_{p}(-t)=-1$ and

$$
W_{t, p}^{*, \prime}\left(1,0, \Phi_{p}\right)=-\frac{1}{2} f_{p} \ell_{p}(t) \log p \cdot W_{t, p}^{*}\left(1,0, \Phi_{p}^{(p)}\right) .
$$

Proof. This is the combination of Corollaries 3.5 and 3.7 of [11].

Proposition 2.2. Let $\tau=u+i v$ be in the upper half plane.

(1) If $t<0$, then $W_{t, \infty}^{*}\left(g_{\tau}, 0, \Phi_{\infty}\right)=0$ and

$$
W_{t, \infty}^{*, \prime}\left(g_{\tau}, 0, \Phi_{\infty}\right)=-\frac{1}{2} \beta_{1}(4 \pi|t| v) \cdot W_{t, \infty}^{*}\left(g_{\tau}, 0, \Phi_{\infty}^{(\infty)}\right)=i \sqrt{v} e^{2 \pi i t \tau} \beta_{1}(4 \pi|t| v) .
$$

(2) If $t=0$, then

$$
W_{0, \infty}^{*}\left(g_{\tau}, 0, \Phi_{\infty}^{(\infty)}\right)=-W_{0, \infty}^{*}\left(g_{\tau}, 0, \Phi_{\infty}\right)=-i \sqrt{v} .
$$


(3) If $t>0$, then $W_{t, \infty}^{*}\left(g_{\tau}, 0, \Phi_{\infty}^{(\infty)}\right)=0$ and

$$
W_{t, \infty}^{*, \prime}\left(\tau, 0, \Phi_{\infty}^{(\infty)}\right)=-\frac{1}{2} \beta_{1}(4 \pi|t| v) \cdot W_{t, \infty}^{*}\left(g_{\tau}, 0, \Phi_{\infty}\right)=-i \sqrt{v} e^{2 \pi i t \tau} \beta_{1}(4 \pi|t| v) .
$$

Here for $t>0$

$$
\beta_{1}(t)=\int_{1}^{\infty} u^{-1} e^{-u t} d u
$$

Proof. The result follows from Lemmas 3.10 and 3.11 of [11].

We now investigate the local Whittaker values at the primes $p \mid N$.

Lemma 2.2. Suppose that $p \mid N$ (i.e., $B$ is ramified at $p$ ), so $p$ is inert in $K$. Then

$$
W_{t, p}^{*}\left(1, s, \Phi_{p}\right)=-\frac{1+p}{p+p^{-s}}+\sum_{r=0}^{v_{p}(t)}\left(-p^{-s}\right)^{r},
$$

and for each integer $t$ with $v_{p}(t)$ even

$$
W_{t, p}^{*}\left(1, s, \Phi_{p}^{(p)}\right)= \begin{cases}0, & \text { if } v_{p}(t)=0 \\ 1, & \text { if } v_{p}(t) \geq 2\end{cases}
$$

Proof. The first statement is [11, Lemma 3.4], see also Yang [12]. It is well known that [11, Lemma 3.1]

$$
\int_{\mathcal{O}_{p}^{\times}} \psi_{p}\left(-b t / p^{r}\right) d b= \begin{cases}1-p^{-1}, & \text { if } r<v_{p}(t)+1 \\ -p^{-1}, & \text { if } r=v_{p}(t)+1 \\ 0, & \text { if } r>v_{p}(t)+1 .\end{cases}
$$

Also recall the following formula regarding the value $\Phi_{p}^{(p)}$ at $k=\left(\begin{array}{ll}a & b \\ c & d\end{array}\right) \in S L_{2}\left(\mathbb{Z}_{p}\right)$ [10, Lemma 3.1.3]

$$
\Phi_{p}^{(p)}(k)= \begin{cases}p^{-2}, & \text { if } v_{p}(c)=0 \\ -p^{-1}, & \text { if } v_{p}(c)=1 \\ 1, & \text { if } v_{p}(c) \geq 2\end{cases}
$$

By the above two identities and suppose $v_{p}(t)$ is even

$$
\begin{aligned}
& W_{t, p}\left(1, s, \Phi_{p}^{(p)}\right) \\
& =\left\{\begin{array}{cl}
p^{-2}-p^{-s-2}, & \text { if } v_{p}(t)=0, \\
p^{-2}+p^{-s-1}\left(1-p^{-1}\right)+\sum_{r=2}^{v_{p}(t)}\left(-p^{-s}\right)^{r} \int_{\mathcal{O}_{p}^{\times}} \psi\left(\frac{-b t}{p^{r}}\right) d b & \\
-p^{-\left(v_{p}(t)+1\right) s} \int_{\mathcal{O}_{p}^{\times}} \psi\left(\frac{-b t}{p^{v^{p}(t)+1}}\right) d b, & \text { if } v_{p}(t) \geq 2 .
\end{array}\right.
\end{aligned}
$$

So $W_{t, p}\left(1,0, \Phi_{p}^{(p)}\right)=0$ provided $v_{p}(t)=0$. Suppose $v_{p}(t) \geq 2$ is even then

$$
W_{t, p}\left(1, s, \Phi_{p}^{(p)}\right)=p^{-2}+p^{-s-1}-p^{-s-2}+\left(1-p^{-1}\right) \sum_{r=2}^{v_{p}(t)}\left(-p^{-s}\right)^{r}+p^{-\left(v_{p}(t)+1\right) s-1} .
$$

Specializing at $s=0$ we get

$$
W_{t, p}\left(1,0, \Phi_{p}^{(p)}\right)=p^{-2}-p^{-2}+p^{-1}+1-p^{-1}+p^{-1}=1+p^{-1}=L\left(1, \omega_{p}\right)^{-1} .
$$


Thus $W_{t, p}^{*}\left(1,0, \Phi_{p}^{(p)}\right)=L\left(1, \omega_{p}\right) \cdot W_{t, p}\left(1,0, \Phi_{p}^{(p)}\right)=1$.

Corollary 2.1. Suppose that $p \mid N$, then

(1) $W_{t, p}^{*}(1,0, \Phi)=0$ if and only if $v_{p}(t)$ is even;

(2) if $v_{p}(t)$ is even then

$$
\begin{aligned}
W_{t, p}^{*, \prime}\left(1,0, \Phi_{p}\right) & =-\left(\frac{1}{1+p}+\frac{v_{p}(t)}{2}\right) \log p \\
& =\left(-\frac{1}{2} f_{p} \ell_{p}(t) \log p-c_{p}^{\prime}(0)\right) \cdot W_{t, p}^{*}\left(1,0, \Psi_{p}^{(p)}\right)
\end{aligned}
$$

see (2.9) for the definition of $c_{p}(s)$;

(3) if $v_{p}(t)$ is even then

$$
W_{t, p}^{*, \prime}\left(1,0, \Phi_{p}\right)+c_{p}^{\prime}(0) \cdot W_{t, p}^{*}\left(1,0, \Psi_{p}^{(p)}\right)=-\frac{1}{2} f_{p} \ell_{p}(t) \log (p) \cdot W_{t, p}^{*}\left(1,0, \Phi_{p}^{(p)}\right)
$$

Proof. (1) is clear. (2) follows by taking the derivative and noticing that (2.9)

$$
c_{p}^{\prime}(0)=\frac{1}{p+1} \log p
$$

and that [11, Corollary 3.2]

$$
W_{t, p}^{*}\left(1,0, \Psi_{p}^{(p)}\right)=1 .
$$

For (3) notice that if $v_{p}(t) \geq 2$, then $(2.19)$ clearly holds because $W_{t, p}^{*}\left(1,0, \Phi_{p}^{(p)}\right)=1$ as well. If $v_{p}(t)=0$, then (2.19) holds trivially because both sides equal zero.

Remark 2.2. The identity (2.19) is the exact motivation for the introduction of the modified Eisenstein series (2.10), also see the proof of Proposition 2.3 for further justification.

Corollary 2.2. Let $t \neq 0$ be an integer, then an odd number of local Whittaker factors $W_{t, p}^{*}\left(1,0, \Phi_{p}\right)$ for $p \leq \infty$ vanish.

Proof. We only give the proof for the case $t>0$, and the case $t<0$ follows similarly. So $W_{t, \infty}^{*}$ does not vanish. Suppose there is an even number of zero local factors, say $W_{t, p_{1}}^{*}, \ldots, W_{t, p_{2 m}}^{*}$. Let $p_{1}, \ldots, p_{k}$ be the ones that do not divide $N$ and let $S=$ $\left\{p_{k+1}, \ldots, p_{2 m}\right\}$ be the part of primes that divide $N$. From results above we know that if $p \mid N$ and $p \notin S$ then $\omega_{p}(-t)=-1$, and

$$
-1=\prod_{p<\infty} \omega_{p}(-t)=\prod_{i=1}^{k} \omega_{p_{i}}(-t) \cdot \prod_{p \mid N, p \notin S} \omega_{p_{j}}(-t) .
$$

Let $d$ be the number of prime divisors of $N$, then $k+[d-(2 m-k)]=2 k+d-2 m$ must be odd, which is a contradiction because $d$ is even.

Now we can relate the non-constant Fourier coefficients of $\mathcal{E}_{t}^{\prime}\left(g_{\tau}, 0, \Phi\right)$ to those of coherent Eisenstein series $E_{t}\left(g_{\tau}, 0, \Phi^{(p)}\right)$ for various primes $p$.

Proposition 2.3. (1) If $t>0$ is an integer then

$$
\mathcal{E}_{t}^{\prime}\left(g_{\tau}, 0, \Phi\right)=-\left(2 w_{K}\right)^{-1} h_{K} \cdot \sum_{p} f_{p} \ell_{p}(t) \log (p) \cdot E_{t}\left(g_{\tau}, 0, \Phi^{(p)}\right),
$$


where $p$ ranges over finite primes that are not split in $K$.

(2) If $t<0$ is an integer then

$$
\mathcal{E}_{t}^{\prime}\left(g_{\tau}, 0, \Phi\right)=-\left(2 w_{K}\right)^{-1} h_{K} \cdot \beta_{1}(4 \pi|t| v) E_{t}\left(g_{\tau}, 0, \Phi^{(\infty)}\right) .
$$

Proof. We only give the proof of part (1). As $t>0$ only $W_{t, p}^{*}\left(1,0, \Phi_{p}\right)$ at non-split primes $p$ can vanish. Since $W_{t, p}^{*}\left(1, s, \Phi_{p}\right)$ is 1 for almost all $p$ we can take the product of local factors without worrying about the convergence. Thus

$$
\begin{aligned}
\mathcal{E}_{t}^{\prime}\left(g_{\tau}, 0, \Phi\right) \\
=E_{t}^{*, \prime}\left(g_{\tau}, 0, \Phi\right)+\sum_{p \mid N} c_{p}^{\prime}(0) E_{t}^{*}\left(g_{\tau}, 0, \Psi^{(p)}\right) \\
=D^{1 / 2} W_{t, \infty}^{*}\left(g_{\tau}, 0, \Phi_{\infty}\right)\left(\sum_{p \nmid N} W_{t, p}^{*,}\left(1,0, \Phi_{p}\right) \prod_{l \neq p} W_{t, l}^{*}\left(1,0, \Phi_{l}\right)\right) \\
+D^{1 / 2} W_{t, \infty}^{*}\left(g_{\tau}, 0, \Phi_{\infty}\right)\left(\sum_{p \mid N} W_{t, p}^{*, \prime}\left(1,0, \Phi_{p}\right) \prod_{l \neq p} W_{t, l}^{*}\left(1,0, \Phi_{l}\right)\right) \\
+D^{1 / 2} W_{t, \infty}^{*}\left(g_{\tau}, 0, \Phi_{\infty}\right) \sum_{p \mid N} c_{p}^{\prime}(0) W_{t, p}^{*}\left(1,0, \Psi_{p}^{(p)}\right) \prod_{l \neq p} W_{t, l}^{*}\left(1,0, \Psi_{l}^{(p)}\right) \\
=D^{1 / 2} W_{t, \infty}^{*}\left(g_{\tau}, 0, \Phi_{\infty}\right)\left(\sum_{p \nmid N}-\frac{1}{2} f_{p} \ell_{p}(t) \log (p) \cdot W_{t, p}^{*}\left(1,0, \Phi_{p}^{(p)}\right) \prod_{l \neq p} W_{t, l}^{*}\left(1,0, \Phi_{l}\right)\right) \\
=-D^{1 / 2} W_{t, \infty}^{*}\left(g_{\tau}, 0, \Phi_{\infty}\right)\left(\sum_{p \mid N} \frac{1}{2} f_{p} \ell_{p}(t) \log (p) \cdot W_{t, p}^{*}\left(1,0, \Phi_{p}^{(p)}\right) \prod_{l \neq p} W_{t, l}^{*}\left(1,0, \Phi_{l}^{(p)}\right)\right) \\
=-\frac{1}{2} \sum_{p}^{1 / 2} f_{p} \ell_{p}(t) \log (p) \cdot E_{t, \infty}^{*}\left(g_{\tau}, 0, \Phi_{\infty}\right)\left(\sum_{p} f_{p} \ell_{p} \log (p) \Phi^{(p)}\right) \\
\left.=-\left(2 w_{K}\right)^{-1} h_{K} \cdot \sum_{p} f_{p} \ell_{p}(t) \log (p) W_{t, p}^{*}\left(1,0, \Phi_{p}^{(p)}\right) \prod_{l \neq p} W_{t, l}^{*}\left(1,0, \Phi_{l}^{(p)}\right)\right) \\
\\
=
\end{aligned}
$$

where we have applied Lemma 2.1, Proposition 2.1 and Corollaries 2.1 and 2.2.

The next proposition is a key result proved in [11, Proposition 3.16], which follows essentially from a Siegel-Weil formula. Let $\mathfrak{a}_{1}=(1), \ldots, \mathfrak{a}_{h_{K}}$ be a set of representatives of $\mathrm{Cl}(K)$. For each prime $p$ non-split in $K$, let $L_{1}^{(p)}=L^{(p)}$ be the quadratic lattice defined by (2.1) (or by (2.2) if $p=\infty$ ), and define

$$
L_{i}^{(p)}=V^{(p)} \cap \mathfrak{a}_{i} \overline{\mathfrak{a}}_{i}^{-1} \widehat{L}^{(p)},
$$

for $i=1, \ldots, h_{K}$. 
Proposition 2.4. Let $\tau=u+i v$ be in the upper plane and $t \neq 0$, then for each (finite or infinite) prime $p$ non-split in $K$

$$
E_{t}\left(g_{\tau}, 0, \Phi^{(p)}\right)=\frac{2}{h_{K}} \sqrt{v} e^{2 \pi i t \tau} \sum_{i=1}^{h_{K}}\left|\left\{y \in L_{i}^{(p)} \mid \mathbf{N}(y)=t\right\}\right|
$$

where $L_{i}^{(p)}$ is the quadratic lattice given in (2.11).

Combining Propositions 2.3 and 2.4 we get the following result about the nonconstant Fourier coefficients of the derivative.

Proposition 2.5. Let $\tau=u+i v$ be in the upper half plane.

(1) If $t>0$ is an integer then

$$
\mathcal{E}_{t}^{\prime}\left(g_{\tau}, 0, \Phi\right)=-w_{K}^{-1} v^{1 / 2} e^{2 \pi i t \tau} \sum_{p} f_{p} \ell_{p} \log (p) \cdot \sum_{i=1}^{h_{K}}\left|\left\{y \in L_{i}^{(p)} \mid \mathbf{N}(y)=t\right\}\right|,
$$

where $p$ ranges over finite primes which are not split in $K$.

(2) If $t<0$ is an integer then

$$
\mathcal{E}_{t}^{\prime}\left(g_{\tau}, 0, \Phi\right)=-w_{K}^{-1} v^{1 / 2} e^{2 \pi i t \tau} \beta_{1}(4 \pi|t| v) \cdot \sum_{i=1}^{h_{K}}\left|\left\{y \in L_{i}^{(\infty)} \mid \mathbf{N}(y)=t\right\}\right| .
$$

Although the constant Fourier coefficient is not our main concern, for the sake of completeness we also sketch the computation of $\mathcal{E}_{0}^{\prime}\left(g_{\tau}, 0, \Phi\right)$.

Proposition 2.6. Let $\tau=u+i v$ be in the upper half plane. Then

$$
\begin{array}{r}
\mathcal{E}_{0}^{\prime}\left(g_{\tau}, s, \Phi\right)=\frac{h_{K} \sqrt{v}}{w_{K}}\left(1+\left(-\frac{1}{2}\right)^{d}\right)\left(\frac{1}{2} \log (v D)+\frac{\Lambda^{\prime}(1, \omega)}{\Lambda(1, \omega)}\right) \\
+\frac{h_{K} \sqrt{v}}{w_{K}} \sum_{p \mid N}\left(\frac{\left(-\frac{1}{2}\right)^{d-1}(p-1)+1}{p+1}\right) \log (p) .
\end{array}
$$

Proof. To apply the results above to the case $t=0$ we just need to take $v_{p}(t)=\infty$ for $p<\infty$ and take the limit. So we have [7, (2.19)] or [11, Proposition 3.14]

$$
\begin{aligned}
& E_{0}^{*}\left(g_{\tau}, s, \Phi\right) \\
& \quad=D^{(s+1) / 2} \Lambda(s+1, \omega) \Phi\left(g_{\tau}, s\right)+D^{(s+1) / 2} M^{*}(s) \Phi\left(g_{\tau}, s\right) \\
& \quad=(v D)^{(s+1) / 2} \Lambda(s+1, \omega)-D^{s / 2} v^{(1-s) / 2} \Lambda(s, \omega) \prod_{p \mid N}\left(-\frac{1+p}{p+p^{-s}}+\frac{1}{1+p^{-s}}\right) .
\end{aligned}
$$

Also at each $p \mid N[11$, Corollary 3.2]

$$
W_{0, p}^{*}\left(1, s, \Psi_{p}^{(p)}\right)=L\left(s, \omega_{p}\right),
$$

which implies that at $s=0$

$$
E_{0}^{*}\left(g_{\tau}, 0, \Psi^{(p)}\right)=(v D)^{1 / 2} \Lambda(1, \omega)+v^{1 / 2} \Lambda(0, \omega)\left(-\frac{1}{2}\right)^{d} .
$$

By the functional equation of $\Lambda(s, \omega)$

$$
D^{s / 2} \Lambda(s, \omega)=D^{(1-s) / 2} \Lambda(1-s, \omega),
$$


we get

So

$$
E_{0}^{*}\left(g_{\tau}, 0, \Psi^{(p)}\right)=v^{1 / 2} \Lambda(0, \omega)\left(1+\left(-\frac{1}{2}\right)^{d}\right)
$$

$$
\begin{aligned}
\mathcal{E}_{0}^{\prime}\left(g_{\tau}, 0, \Phi\right)=E_{0}^{*,} & \left(g_{\tau}, 0, \Phi\right)+\sum_{p \mid N} c_{p}^{\prime}(0) E_{0}^{*}\left(g_{\tau}, 0, \Psi^{(p)}\right) \\
= & \frac{1}{2} \log (v D)(v D)^{1 / 2} \Lambda(1, \omega)+(v D)^{1 / 2} \Lambda^{\prime}(1, \omega) \\
& -\frac{1}{2} v^{1 / 2} \log (D / v) \Lambda(0, \omega)(-1 / 2)^{d}-v^{1 / 2} \Lambda^{\prime}(0, \omega)(-1 / 2)^{d} \\
& +v^{1 / 2} \Lambda(0, \omega)\left(-\frac{1}{2}\right)^{d} \sum_{p \mid N} \frac{p-3}{2(p+1)} \log (p) \\
& +v^{1 / 2} \Lambda(0, \omega)\left(1+\left(-\frac{1}{2}\right)^{d}\right) \sum_{p \mid N} \frac{1}{p+1} \log (p)
\end{aligned}
$$

Note that

$$
\Lambda^{\prime}(0, \omega)=-D^{1 / 2}\left(\log (D) \Lambda(1, \omega)+\Lambda^{\prime}(1, \omega)\right)
$$

therefore

$$
\begin{aligned}
\mathcal{E}_{0}^{\prime}\left(g_{\tau}, 0, \Phi\right)= & (v D)^{1 / 2} \Lambda(1, \omega)\left(1+\left(-\frac{1}{2}\right)^{d}\right)\left(\frac{1}{2} \log (v D)+\frac{\Lambda^{\prime}(1, \omega)}{\Lambda(1, \omega)}\right) \\
& +(v D)^{1 / 2} \Lambda(1, \omega) \sum_{p \mid N}\left(\frac{\left(-\frac{1}{2}\right)^{d-1}(p-1)+1}{p+1}\right) \log (p),
\end{aligned}
$$

which together with the class number formula $\Lambda(1, \omega)=\frac{h_{K}}{w_{K} D}$ concludes the proof of $(2.21)$.

\section{Geometric computations}

In this section, we first introduce the moduli scheme $M$ of QM abelian surfaces with an extra action by $\mathcal{O}_{K}$, called with $\mathrm{CM}$ by $\mathcal{O}_{K}$. Then for each positive integer $t$ we define a zero-dimensional moduli scheme $Z(t)$ which has a natural morphism to $M$. In the end, we will compute the degree of $Z(t)$, compare it with the $t$-th Fourier coefficient of $\mathcal{E}^{\prime}\left(g_{\tau}, 0, \Phi\right)$, and then prove (1.1).

3.1. A moduli scheme. An abelian surface $A$ over a base scheme $S$ is said to have quaternionic multiplication $(\mathrm{QM})$ by $\mathcal{O}_{B}$ if there is an embedding $\imath: \mathcal{O}_{B} \rightarrow \operatorname{End}_{S}(A)$, such that the action of $\mathcal{O}_{B}$ is "special" in the sense of Drinfeld. Since the exact definition of "special" will not be used, we simply skip its definition and refer readers to $\left[5\right.$, p. 5]. We say a $\mathrm{QM}$ abelian surface $A$ has $\mathrm{CM}$ by $\mathcal{O}_{K}$ if there is an embedding

$$
\iota: \mathcal{O}_{K} \rightarrow \operatorname{End}(A, \imath),
$$

where $\operatorname{End}(A, \imath)$ means the subring of endomorphisms of $A$ which commute with the action of $\mathcal{O}_{B}$. 
We consider the following moduli problem $\mathcal{M}$ on the category $\left(\mathrm{Sch} / \mathcal{O}_{K}\right)$. To each scheme $S$ over $\mathcal{O}_{K}$, let $\mathcal{M}(S)$ be the set of isomorphism classes of triples $(A, \imath, \iota)$, where $(A, \imath)$ is a QM abelian surface over $S$, and $\iota: \mathcal{O}_{K} \rightarrow \operatorname{End}(A, \imath)$ is the CM such that the induced homomorphism $\operatorname{Lie}(\iota): \mathcal{O}_{K} \rightarrow \operatorname{End}_{\mathcal{O}_{S}, B}(\operatorname{Lie} A)=\mathcal{O}_{S}$ coincides with the structure homomorphism of $\mathcal{O}_{S}$.

The following result on the functor $\mathcal{M}$ is well known, see $[5,13]$ and an argument in [7, Proposition 5.1] for the case of CM elliptic curves.

Theorem 3.1. The moduli problem $\mathcal{M}$ has a coarse moduli space $M$ which is étale over $\operatorname{Spec}\left(\mathcal{O}_{K}\right)$.

The existence of the coarse moduli scheme $M$ follows by the relative representability of the natural morphism $(A, \imath, \iota) \mapsto(A, \imath)$ from $\mathcal{M}$ to the algebraic stack of QM abelian surfaces. The étaleness of $\mathcal{M}$ over $\operatorname{Spec}\left(\mathcal{O}_{K}\right)$ can be easily obtained by Gross' work on canonical liftings and the Serre-Tate theorem on $p$-divisible groups of abelian schemes. The étaleness of the moduli scheme $M$ is due to the fact that the automorphism group of each geometric point $\xi$ on $\mathcal{M}$ is $\mathcal{O}_{K}^{\times}$, which acts trivially on the completion $\widehat{\mathcal{O}}_{\mathcal{M}, \xi}$.

Actually the coarse moduli scheme $M$ consists of $2^{d}$ disjoint copies of $\operatorname{Spec}\left(\mathcal{O}_{H}\right)$, where $d$ as usual is the number of prime factors of $N$ and $H$ is the Hilbert class field of $K$. Each copy is distinguished by the orientation class determined by the $\mathcal{O}_{K}$-action on the complex QM-abelian surface. Here the orientation class attached to $(A, \imath, \iota) \in \mathcal{M}(\mathbb{C})$ is determined as follows. For each finite prime $l$ let $T_{l}(A)$ be the $l$-adic Tate module of $A$. Then $T_{l}(A)$ is a free $\mathcal{O}_{B}$-module of rank 1 , and thus $\iota$ induces a natural embedding

$$
\iota_{l}: \mathcal{O}_{K, l} \rightarrow \operatorname{End}_{B}\left(T_{l}(A)\right) \cong \mathcal{O}_{B, l}^{\text {opp }},
$$

see [5, Lemma 2.7] for the last isomorphism. The group $\mathcal{O}_{B, l}^{\text {opp }, \times}$ acts on the set of embeddings $\operatorname{Hom}\left(\mathcal{O}_{K, l}, \mathcal{O}_{B, l}^{\text {opp }}\right)$ through conjugation. The class $\bar{\iota}_{l}$ of $\iota_{l}$ in the coset $\operatorname{Hom}\left(\mathcal{O}_{K, l}, \mathcal{O}_{B, l}^{\text {opp }}\right) / \mathcal{O}_{B, l}^{\text {opp }, \times}$ is called the orientation (class) of $(A, \imath, \iota)$ at $l$. The collection $\left\{\bar{\iota}_{l}\right\}_{l}$ over all finite primes $l$ is called the orientation of $(A, \imath, \iota)$. Locally at each $l$, $\operatorname{Hom}\left(\mathcal{O}_{K, l}, \mathcal{O}_{B, l}^{\text {opp }}\right) / \mathcal{O}_{B, l}^{\text {opp }, \times}$ has cardinality 2 or 1 depending on whether or not $l \mid N$, hence there are exactly $2^{d}$ different orientation classes, see $[13$, p. 54$]$ or $[5$, p. 11] for more details. By CM theory (Shimura-Taniyama) each orientation determines one copy of Spec $\left(\mathcal{O}_{H}\right)$, whose points over any algebraically closed are permuted by the Galois group $\operatorname{Gal}(H / K) \cong \mathrm{Cl}(K)$, see [13, p. 55]. Altogether there are exactly $2^{d}$ disjoint copies of $\mathcal{O}_{H}$ in $M$.

To conclude this section we remark that the orientation introduced above is parallel to the one on CM elliptic curves with a cyclic $M$-structure. In the elliptic curve case, an orientation is equivalent to choosing an $\mathcal{O}_{K}$-ideal $\mathcal{M}$ such that $\mathcal{O}_{K} / \mathcal{M} \cong \mathbb{Z} / M \mathbb{Z}$, see $[3]$.

3.2. Special endomorphisms and zero cycles. We first give the following definition of special endomorphisms on a QM abelian surface with CM by $\mathcal{O}_{K}$.

Definition 3.1. Let $(A, \imath, \iota) \in \mathcal{M}(S)$. A special endomorphism of $(A, \imath, \iota)$ is an element $y \in \operatorname{End}(A, \imath)$ such that

$$
y \cdot \iota(a)=\iota(\bar{a}) \cdot y,
$$

for all $a \in \mathcal{O}_{K}$. 
In general, the set of special endomorphisms of $(A, \imath, \iota) \in \mathcal{M}(S)$ is denoted in short by $L(A)$. If $p$ is a finite prime, the set of special endomorphisms of $(A, \imath, \iota) \in M\left(\overline{\mathbb{F}}_{p}\right)$ is also denoted by $L^{(p)}(A)$, which is a non-trivial if and only if $p$ is not split in $K$.

Definition 3.2. Let $\mathcal{Z}(t)$ denote the functor which assigns to each $\mathcal{O}_{K}$-scheme $S$ the set of isomorphism classes of quadruples $(A, \imath, \iota, y)$, where $(A, \imath, \iota) \in \mathcal{M}(S)$ and $y \in L(A)$ such that

$$
\mathbf{N}(y)=y \cdot y^{\prime}=t .
$$

Here $y^{\prime}$ is the main involution of $\operatorname{End}_{B}^{0}(A)$ which is either $K$ or a quaternion algebra containing $K$.

The natural forgetful morphism pr: $(A, \imath, \iota, y) \mapsto(A, \imath, \iota)$ from $\mathcal{Z}(t)$ to $\mathcal{M}$ is relatively representable. So $\mathcal{Z}(t)$ has a coarse moduli scheme, which is denoted by $Z(t)$. Similar to the CM elliptic curve case the scheme $Z(t)$ is artinian and concentrated on finite characteristics $p$ which are not split in $K$. The degree of $Z(t)$ is defined as

$$
\operatorname{deg}(Z(t))=\sum_{\xi \in Z(t)} \log \left(\left|\mathcal{O}_{Z(t), \xi}\right|\right)
$$

where the sum is over the geometric points of $Z(t)$.

Recall the following theorem about the length of $\mathcal{O}_{Z(t), \xi}$ at each geometric point $\xi$, which is due to Gross [1]. Let $p$ be non-split in $K$ and let $\mathfrak{p}$ be the prime ideal over $p$ in $K$. Let $k(\mathfrak{p})=\mathcal{O}_{K} / \mathfrak{p}$ be the residue field of $\mathfrak{p}$.

Proposition 3.1 (Gross). Let $d_{p}=v_{p}(D)$ and $f_{p}$ be the residue degree of $p$ in $K$. Then

$$
\operatorname{length}_{\mathcal{O}_{K, \mathfrak{p}}} \mathcal{O}_{Z(t), \xi}=\ell_{p}(t)
$$

where

$$
\ell_{p}(t)=\frac{v_{p}(t)+d_{p}-1-\epsilon_{p}}{f_{p}}+1
$$

and

$$
\epsilon_{p}= \begin{cases}1, & \text { if } B \text { is ramified at } p, \text { i.e., } p \mid N \\ 0, & \text { if } B \text { is unramified at } p .\end{cases}
$$

As

$$
|Z(t)(\overline{k(\mathfrak{p})})|=\sum_{(A, \imath, \iota) \in M(\overline{k(\mathfrak{p})})}\left|\left\{y \in L^{(p)}(A) \mid \mathbf{N}(y)=t\right\}\right|,
$$

the next lemma on $\operatorname{deg}(Z(t))$ is prompt.

Lemma 3.1. The degree of $Z(t)$ is given by

$$
\operatorname{deg}(Z(t))=2^{d} \sum_{p} f_{p} \ell_{p}(t) \log (p) \cdot \sum_{i=1}^{h_{K}}\left|\left\{x \in L\left(A_{i}\right) \mid \mathbf{N}(x)=t\right\}\right|,
$$

where $p$ ranges over primes which are not split in $K$, and $\left(A_{i}, \imath_{i}, \iota_{i}\right)$ for $i=1, \ldots, h_{K}$ runs over points in $M(\overline{k(\mathfrak{p})})$ with a fixed orientation. 
For all $i=1, \ldots, h_{K}, \operatorname{End}\left(A_{i}, \imath_{i}\right)$ is an order of discriminant $N p$ in $B^{(p)}$, and $\mathcal{O}_{K}$ is optimally embedded into $\operatorname{End}\left(A_{i}, \imath_{i}\right)$. By the Chevalley-Hasse-Noether theorem (see $\left[2\right.$, Proposition 3.4]) there is an ideal $\mathfrak{a}_{i}$ such that

$$
L^{(p)}\left(A_{i}\right)=\mathfrak{a}_{i}{\overline{\mathfrak{a}_{i}}}^{-1} L^{(p)}\left(A_{1}\right) .
$$

Conversely, by canonical lifting theory [1] and Serre-Tate theorem on $p$-divisible groups of abelian schemes, every ideal class $\mathfrak{a}_{i}$ shows up. We can choose $\mathfrak{a}_{1}=(1), \ldots, \mathfrak{a}_{h_{K}}$ to be the same set of representatives as in Proposition 2.4. Then $L_{i}^{(p)}=L^{(p)}\left(A_{i}\right)$ (see (2.20) for the construction of $\left.L_{i}^{(p)}\right)$ and for each finite prime $p$ non-split in $K$

$$
\sum_{i=1}^{h_{K}}\left|\left\{y \in L_{i}^{(p)} \mid \mathbf{N}(y)=t\right\}\right|=\sum_{i=1}^{h_{K}}\left|\left\{y \in L^{(p)}\left(A_{i}\right) \mid \mathbf{N}(y)=t\right\}\right| .
$$

For each negative integer $t$ and $\tau=u+i v$ in the upper half plane, we define an Arakelov divisor concentrated at archimedean points $M(\mathbb{C})$ of the scheme $M$ (notice that $Z(t)$ is actually not a subscheme of $M)$. For a point $(A, \imath, \iota)$ of $M(\mathbb{C})$, write $A^{a}$ for the underlying real torus. Let $\operatorname{End}\left(A^{a}, \imath\right)$ denote the ring of real analytic endomorphisms of $A^{a}$ which commute with the action of $\mathcal{O}_{B}$, then $\operatorname{End}\left(A^{a}, \imath\right) \cong \mathcal{O}_{B}^{\text {opp }}$ [5, Lemma 2.7]. The set of special endomorphisms (at infinity) of $(A, \imath, \iota)$ is defined as

$$
L^{(\infty)}(A)=\left\{y \in \operatorname{End}\left(A^{a}, \imath\right) \mid y \cdot \iota(a)=\iota(\bar{a}) \cdot y\right\} .
$$

Similar to (3.4) we have

$$
\sum_{i=1}^{h_{K}}\left|\left\{y \in L_{i}^{(\infty)} \mid \mathbf{N}(y)=t\right\}\right|=\sum_{i=1}^{h_{K}}\left|\left\{y \in L^{(\infty)}\left(A_{i}\right) \mid \mathbf{N}(y)=t\right\}\right| .
$$

The Arakelov divisor $Z(t, v)$ is now defined by

$$
Z(t, v)=\sum_{i, j} r_{i}(t, v) \sigma_{i}^{j}
$$

where $\sigma_{i}^{j}$ runs over archimedean places of $M$ with $i=1, \ldots, h_{K}, j=1, \ldots, 2^{d}$ and

$$
r_{i}(t, v)=\beta_{1}(4 \pi|t| v) \cdot\left|\left\{y \in L^{(\infty)}\left(A_{i}\right) \mid \mathbf{N}(y)=t\right\}\right| .
$$

The degree of the Arakelov divisor $Z(t, v)$ is then given by

$$
\operatorname{deg}(Z(t, v))=2^{d} \sum_{i=1}^{h_{K}} r_{i}(t, v)
$$

3.3. Conclusion. We conclude our paper with the following main result.

Theorem 3.2. Let $\tau=u+i v$ be in the upper half plane and let

$$
\phi(\tau)=-\left.\frac{d}{d s}\left(\sqrt{v}^{-1} \mathcal{E}\left(g_{\tau}, s, \Phi\right)\right)\right|_{s=0} .
$$

Then the non-holomorphic modular form $\phi(\tau)$ has the following Fourier expansion

$$
\phi(\tau)=a_{0}(v)+\sum_{t>0} a_{t} e^{2 \pi i t \tau}+\sum_{t<0} a_{t}(v) e^{2 \pi i t \tau},
$$


where

$$
\begin{aligned}
a_{0}(v)= & \frac{h_{K}}{w_{K}}\left(1+\left(-\frac{1}{2}\right)^{d}\right)\left(\frac{1}{2} \log (v D)+\frac{\Lambda^{\prime}(1, \omega)}{\Lambda(1, \omega)}\right) \\
& +\frac{h_{K}}{w_{K}} \sum_{p \mid N}\left(\frac{\left(-\frac{1}{2}\right)^{d-1}(p-1)+1}{p+1}\right) \log (p),
\end{aligned}
$$

for $t>0$

and for $t<0$

$$
a_{t}=\left(2^{d} w_{K}\right)^{-1} \operatorname{deg}(Z(t))
$$

$$
a_{t}(v)=\left(2^{d} w_{K}\right)^{-1} \operatorname{deg}(Z(t, v)) .
$$

Proof. The formula for $a_{0}(v)$ is proved in Proposition 2.6 (2.21). The claim for $a_{t}$ with $t>0$ follows from Proposition 2.5, Lemma 3.1 and (3.4). The claim for $a_{t}(v)$ is obtained by comparing Proposition 2.5, (3.5), (3.6) and (3.7).

Remark 3.1. (1) It should be noted that the construction of $\mathcal{E}\left(g_{\tau}, s, \Phi\right)$ and thus Theorem 3.2 depends on both the constant $\kappa$ and the quaternion algebra $B$.

(2) We begin with a fixed choice of the maximal order $\mathcal{O}_{B}$ of $B$ for which $\mathcal{O}_{K}$ admits an optimal embedding into. By the Chevalley-Hasse-Noether theorem, any two such maximal orders are conjugate by an element of $K_{\mathbb{A}}^{\times}$, which locally on $V$ or $V^{(p)}$ (see Section 2.1) is equivalent to the multiplication by an element from $S O(V)$ or $S O\left(V^{(p)}\right)$. On the other hand, all the Eisenstein series are actually $O(V)$ or $O\left(V^{(p)}\right)$ invariant [6, p. 554], therefore Theorem 3.2 does not depend on the choice of $\mathcal{O}_{B}$.

\section{Acknowledgments}

We would like to thank the referee for valuable comments.

\section{References}

[1] B. Gross, On canonical and quasicanonical liftings, Invent. Math. 84(2) (1986), 321-326.

[2] _ Local orders, root numbers, and modular curves, Amer. J. Math. 110(6) (1988), 11531182.

[3] B. Gross and D. Zagier, Heegner points and derivatives of L-series, Invent. Math. 84(2) (1986), $225-320$.

[4] H. Jacquet and R. P. Langlands, Automorphic forms on GL(2), Springer-Verlag, Berlin, Vol. 114, Lecture Notes Math., 1970.

[5] K. Keating and D. Roberts, Intersection numbers of Heegner divisors on Shimura curves, Pure Appl. Math. Q. 4(4) (2008), part 1, 1165-1204.

[6] S. Kudla, Central derivatives of Eisenstein series and height pairings, Ann. of Math. (2) 146(3) (1997), 545-646.

[7] S. Kudla, M. Rapoport and T. Yang, On the derivative of an Eisenstein series of weight one, Int. Math. Res. Not. 7(7) (1999), 347-385.

[8] _ Derivatives of Eisenstein series and Faltings heights, Compos. Math. 140(4) (2004), $887-951$.

[9] S. Kudla and T. Yang, On the pullback of an arithmetic theta function, 2011, p. 36, arXiv:1106.4732.

[10] H. Xue, Central values of L-functions over CM fields, J. Number Theory 122(2) (2007), 342378.

[11] _ The derivative of an incoherent Eisenstein series, Trans. Amer. Math. Soc. 364 (2012), $3311-3327$. 
[12] T. Yang, CM number fields and modular forms, Pure Appl. Math. Q. 1(2) (2005), part 1, 305-340.

[13] S. Zhang, Heights of Heegner points on Shimura curves, Ann. of Math. (2) 153(1) (2001), $27-147$.

Department of Mathematical Sciences Clemson University Clemson, SC 29634 USA

E-mail address: huixue@clemson.edu 
